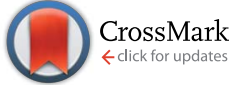

Cite this: RSC Adv., 2017, 7, 14939

Received 3rd January 2017 Accepted 2nd March 2017

DOI: $10.1039 / \mathrm{c} 7 \mathrm{ra00051 \textrm {k }}$

rsc.li/rsc-advances

\title{
Encapsulation of fish oil in a coaxial electrospun nanofibrous mat and its properties
}

\author{
Huan Yang, $\dagger^{\mathrm{a}}$ Peng Wen, $\dagger^{\mathrm{a}}$ Kun Feng, ${ }^{\mathrm{a}}$ Min H. Zong, ${ }^{a}$ Wen Y. Lou ${ }^{\mathrm{a}}$ and Hong Wu $\mathrm{W}^{* a b}$ \\ Coaxial electrospinning was firstly developed to encapsulate fish oil in composite nanofibers to improve its \\ oxidative stability. TEM analysis revealed that a clear core-shell structure was formed in the obtained coaxial \\ nanofibers, the average diameter of which was $560 \mathrm{~nm}$. The loading capacity and encapsulation efficiency \\ of fish oil were $14.5 \%$ and $96.9 \%$, respectively. FTIR data demonstrated that fish oil was successfully \\ entrapped into the coaxial electrospun nanofibers. The oxidative stability of encapsulated fish oil in the \\ coaxial nanofibers was significantly enhanced compared to that in single nanofibers. Meanwhile, most of \\ the encapsulated fish oil can be efficiently released from the coaxial electrospun nanofibers and the \\ release was controlled by a combination of diffusion and macromolecular chain relaxation. This coaxial \\ electrospun nanofibrous mat with favourable oxidative stability and release properties has potential for \\ an application as a nutritional additive.
}

\section{Introduction}

Fish oil is rich in essential polyunsaturated fatty acids, such as eicosapentaenoic acid (EPA) and docosahexaenoic acid (DHA), which can serve as a functional food and is helpful in maintaining good health and in the prevention of a range of human diseases. ${ }^{1,2}$ Despite the positive health benefits of fish oil, its application as a food nutritional additive faces some challenges. One of the major challenges is that the high degree of unsaturated carbon-carbon double bonds in fish oil makes it easily susceptible to oxidation, thus it is essential to improve its oxidative stability. In this respect, encapsulation techniques have been considered for protecting fish oil against oxidation and enhancing their bioavailability. The most common approaches for encapsulation are spray drying, liposome entrapment, etc. ${ }^{3,4}$ However, these methods typically produce particles of micro and nano sizes, which may have some potential toxic effects on human health. ${ }^{5}$

Electrospinning has been considered as a significant breakthrough and a promising technology that produces continuous polymer fibers with nanometer sizes. ${ }^{6,7}$ The electrospun nanofibers have many structural and functional advantages, such as a large surface-to-volume ratio, high encapsulation efficiency and great stability of encapsulated bioactive compounds. ${ }^{\mathbf{8 9}}$ Presently, electrospinning has been applied in the fields of tissue engineering, drug delivery systems, textiles and filtration, etc. ${ }^{10,11}$ However, the

${ }^{a}$ School of Food Science and Engineering, South China University of Technology, Guangzhou 510640, China. E-mail: bbhwu@scut.edu.cn; Tel: +86-20-22236669

${ }^{b}$ Guangdong Province Key Laboratory for Green Processing of Natural Products and Product Safety, Guangzhou 510640, China

$\dagger$ This authors have the same contribution and are co-first author. potential of this technique is less explored in the food science field, especially in the production of functional foods. The most commonly used method in encapsulation of bioactive compound is single electrospinning. ${ }^{4}$ Recently, with the development of electrospinning techniques, coaxial electrospinning, which is capable of producing continuous double layer nanofibers by coelectrospinning two materials through a facile one-step procedure, has emerged as an alternative to encapsulate bioactive compounds. ${ }^{12}$ It was reported that olive leaf extract was successfully encapsulated into coaxial silk fibroin/hyaluronic acid nanofibers, which exhibited favourable antibacterial properties and release stability. ${ }^{13}$ Lópezrubio et al. found that coaxial electrospinning was a more efficient method to entrap living bifidobacteria cells and improve their viability compared with single electrospinning. ${ }^{\mathbf{1 4}}$

Zein is the major storage protein of corn, and has extensive application in encapsulation of bioactive substances. To date, there is only one report referring to the encapsulation of fish oil in the zein nanofibers via electrospinning. ${ }^{15}$ It was found that the oxidative stability of encapsulated fish oil in the electrospun zein film was improved compared with non-encapsulated fish oil. However, use of coaxial electrospinning for encapsulation of fish oil to improve its oxidative stability has not been reported yet. Therefore, zein was chosen as the core material, and polyvinylpyrrolidone (PVP), an important synthetic polymer with biocompatible and nontoxic properties, was selected as the filament-forming matrix of the shell part for encapsulation of fish oil. The morphology of the resulting coaxial electrospun nanofibers was characterized by scanning electron microscopy (SEM), transmission electron microscope (TEM), Fourier transform infrared spectroscopy (FTIR) and thermogravimetric analysis (TGA). The oxidative stability of encapsulated fish oil in the coaxial 
nanofibers was investigated and compared with that in the single nanofibers. The shelf life of encapsulated fish oils in both coaxial and single nanofibers were further determined based on their peroxide values. In addition, the release behavior of encapsulated fish oil during an in vitro digestion was investigated.

\section{Materials and methods}

\subsection{Materials}

Zein was purchased from Tokyo Chemical Industry, Tokyo, Japan. Fish oil was a kind gift from Sinomega Biotech Engineering Co., Ltd (Zhejiang, China). PVP was supplied by Jingke Chemical Regent Company (Shanghai, China). Pepsin and trypsin were from Aladdin Chemistry Co., Ltd. (Shanghai, China). Other chemicals and solvents were purchased from Sinopharm Chemical Reagent Co., Ltd. (Guangzhou, China). All materials were used as received without further purification.

\subsection{Electrospinning}

2.2.1 Preparation of electrospinning solution. The core electrospinning solution was prepared by dissolving $25 \mathrm{~g}$ zein in $100 \mathrm{~mL} 80 \%$ (v/v, volume of ethanol/(volume of ethanol + volume of water)) aqueous ethanol at room temperature under magnetic stirring for $0.5 \mathrm{~h}$. Then, $30 \%$ (w/w, weight of oil/weight of zein) fish oil was added to the above zein solution by constant stirring for $1 \mathrm{~h}$. After that, $10 \%$ (w/w, weight of glycerol/weight of zein) glycerol as a plasticizer was added to the above-mentioned solution and mixed until complete homogenization. The shell electrospinning solution was prepared by dissolving PVP (27-31\%, w/v) in $100 \mathrm{~mL}$ aqueous ethanol $(80-100 \%, \mathrm{v} / \mathrm{v})$ under constant magnetic stirring for $0.5 \mathrm{~h}$. Before loading into a syringe, all solutions were left until no air bubble in the liquid phase was observed.

2.2.2 Electrospinning process. The apparatus for electrospinning was developed in-house. A homemade concentric spinneret was used to conduct the coaxial electrospinning process, which equipped with a 22 gauge inner steel needle and an 18 gauge outer steel needle. The core and shell electrospinning solutions were individually loaded in a $10 \mathrm{~mL}$ syringe which connected to the coaxial spinneret. Two syringe pumps (NE-300, New Era Pump Systems Inc., USA), a high voltage power supply (AU Series, Matsusada Precision Inc.) and a grounded collecting plate covered by a piece of aluminum foil were used for the coaxial electrospinning. The flow rates of core and shell electrospinning solutions were controlled at $0.4 \mathrm{~mL}$ $\mathrm{h}^{-1}$ and $0.2 \mathrm{~mL} \mathrm{~h}^{-1}$ respectively. $16 \mathrm{kV}$ voltage was applied to the metal needle tip. The distance from the needle tip to collector was $14 \mathrm{~cm}$. All electrospinning processes were carried out at $24 \pm 3{ }^{\circ} \mathrm{C}$ and a relative humidity of $45 \pm 5 \%$. For comparison, single nanofibrous mat was also produced by a one-fluid single electrospinning of the core solution, which was implemented through adjusting the flow rate of the shell fluid to $0 \mathrm{~mL} \mathrm{~h}^{-1}$ under the same conditions.

\subsection{Characterization and measurements}

A scanning electron microscope (SEM) (EVO18, Oberkochen, Germany) at an accelerating voltage of $10 \mathrm{kV}$ was used to investigate the morphology of the electrospun fibers. Prior to the examination, the samples were left at ambient temperature $\left(24 \pm 3{ }^{\circ} \mathrm{C}\right)$ overnight and then coated with Pt using a sputter coater (K550, Emitech, UK) under vacuum to render them electrically conductive. The fiber diameter distribution was calculated by analysis of around 100 fibers from the SEM image.

Transmission electron microscope (TEM) (JEM-2100F, Tokyo, Japan) observation was carried out to determine the core/shell structure of the coaxial nanofibers. The TEM sample was collected by directly depositing the as-spun nanofibers onto copper grid.

Infrared spectra of samples were collected by using a FTIR spectrometer (Model Equinox 55; Bruker Co., Ettlingen, Germany). Zein, fish oil and other samples were scanned from 500 to $4000 \mathrm{~cm}^{-1}$. Each measurement was an average of 16 scans at $4 \mathrm{~cm}^{-1}$ resolution. The signals were processed using the OPUS spectroscopic software.

The thermal stability of different samples was evaluated by TGA (Q500, TA Instruments, USA). TGA measure changes in the weight of samples as a function of temperature and/or time by heated samples from room temperature to $700{ }^{\circ} \mathrm{C}$ at constant heating rate of $25{ }^{\circ} \mathrm{C} \mathrm{min}^{-1}$ under nitrogen atmosphere (flow rate: $20 \mathrm{~mL} \min ^{-1}$ ).

\subsection{Loading capacity and encapsulation efficiency}

The loading capacity (LC) and encapsulation efficiency (EE) were determined by measuring the amount of nonencapsulated fish oil according to Moomand and Lim (2014) with some modifications. ${ }^{16}$ The electrospun nanofibrous mat (90 $\mathrm{mg}$ ) was submerged in hexane $(8 \mathrm{~mL}$ ) for $1 \mathrm{~min}$ to remove the surface oil. The mixture was filtered and the absorbance of the liquid was determined using a UV-Vis spectrophotometer (UV-2550, Shimadzu, Japan) at $260 \mathrm{~nm}$. The calibration curve was prepared by measuring the absorbance of a series of solutions with different concentrations of fish oil dissolved in hexane. The fish oil concentration-absorbance calibration curve was followed equation $Y=2.493 X+0.213\left(R^{2}=0.992\right.$, wherein $X$ was the concentration of fish oil $\left(\mathrm{mg} \mathrm{mL}^{-1}\right)$, and $Y$ was the absorbance at $260 \mathrm{~nm}$ ). The amount of fish oil in the coaxial nanofibers was then calculated according to this calibration curve. The LC and EE values were calculated as:

$$
\begin{aligned}
& \mathrm{LC}=(A-B) / C \times 100 \\
& \mathrm{EE}=(A-B) / A \times 100
\end{aligned}
$$

where $A$ was the total theoretical amount of fish oil, $B$ was the free amount of fish oil in the collection solution, and $C$ was the weight of the nanofibrous mat.

\subsection{Oxidative stability analysis}

The oxidative stability of the non-encapsulated and encapsulated fish oil was tested under both aerobic and anaerobic conditions at 25, 45 and $60{ }^{\circ} \mathrm{C}$. For the stability test under aerobic condition, approximately $1 \mathrm{~g}$ of each sample was placed in an open Erlenmeyer flask, then kept in dark at 25, 45 and 
$60{ }^{\circ} \mathrm{C}$ and taken for analysis at day $0,1,2,3,4$ and 5 . Under the anaerobic condition, the treatment was similar to that of aerobic experiment, except all the samples were sealed in Erlenmeyer flasks filled with nitrogen and taken for analysis at day $0,5,10,15,20$ and 25. Lipid oxidation of the samples were evaluated by testing their lipid hydroperoxides following a FOX (ferrous oxidation in xylenol orange) method according to Moomand and Lim (2014). ${ }^{16}$

\subsection{Release behavior of encapsulated fish oil}

The in vitro fish oil release profiles of nanofibers were determined as follows: the sample was placed into serum bottle containing $15 \mathrm{~mL}$ release medium and incubated in a rotation shaker at $100 \mathrm{rpm}$ and $37^{\circ} \mathrm{C}$. The release media were simulated gastric fluid (SGF) ( $\mathrm{HCl}$ solution, $\mathrm{pH}$ 1.2) and simulated intestinal fluid (SIF) (phosphate buffer, pH 6.8), prepared according to the Chinese Pharmacopoeia 2005, respectively. To determine the effect of enzyme on the release behavior, $2 \%$ pepsin and trypsin were added to the SGF and SIF, respectively.

In the release behavior experiment in separate SGF or SIF, the nanofibrous mat was incubated in SGF or SIF. Aliquot of 1 $\mathrm{mL}$ of the sample was collected at predetermined time interval from the solution.

In the release behavior experiment in sequential SGF and SIF treatment, the nanofibrous mat was incubated in SGF for $2 \mathrm{~h}$ firstly, and then transferred to SIF for $4 \mathrm{~h}$. An aliquot $(1 \mathrm{~mL})$ of the sample was collected at predetermined time interval from the solution.

Similar to the LC and EE measurement of fish oil, the concentration of fish oil was measured spectrophotometrically at $260 \mathrm{~nm}$ following the same methodology as described at 2.4.

\subsection{Statistical analysis}

SPSS software (Version 18.0, Chicago, USA) was used to analyze the resulting data. All experiments were performed at least in triplicate. Statistical significance among treatments was evaluated by analysis of variance (One-way ANOVA with Duncan test). The probability of test statistic $(p)$ was set at 0.05 .

\section{Results and discussion}

\subsection{Fabrication of nanofibrous mat by coaxial electrospinning}

Previous studies showed that the concentration of solvent and polymer determined if the electrospinning solution can be electrospun into nanofibers, which also had remarkable effect on the morphology of nanofibers. ${ }^{17,18}$ Here, the shell electrospinning solution was prepared by dissolving PVP in aqueous ethanol. Fig. 1 and 2 summarized the SEM images of the electrospun PVP nanofibers at different concentration of ethanol and PVP, respectively. As shown in Fig. 1, it was difficult to form distinct nanofibers at low ethanol concentration (80\%), but high concentration of ethanol would favor the production of PVP nanofibers, especial for pure ethanol. The possible reason was that the slow evaporation rate of ethanol at low concentration made it difficult for nanofibers to completely dry before
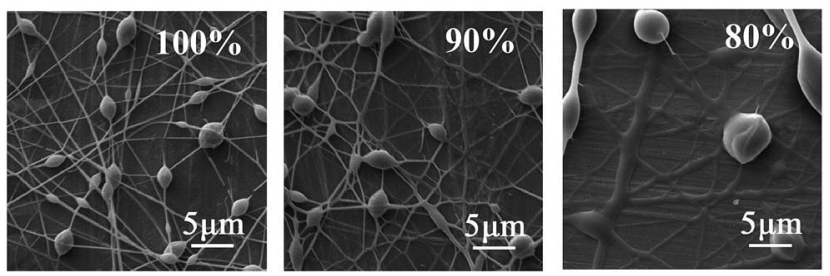

Fig. 1 SEM images of the PVP nanofibers at different ethanol concentration.

it hit the collector. As depicted in Fig. 2, smoother nanofibers were obtained when the concentration of PVP increased from $27 \%$ to $31 \%(\mathrm{w} / \mathrm{v})$ due to the more dominant chain entanglement of high concentration of PVP which stabilized the polymer jet. ${ }^{19}$ Therefore, 31\% PVP which dissolved in pure ethanol was chosen as shell solution for coaxial electrospinning.

TEM image was used to reveal the core-shell structure of the resulting electrospun nanofibers. For comparison, the single nanofiber was also analyzed, which produced by single electrospinning of the core solution. As shown in Fig. 3, compared with the TEM image of single nanofiber, the interface between the core and shell layers was clearly observed for the TEM image of coaxial nanofiber. It was due to that zein (core) is denser than PVP (shell), thus fewer electrons were transmitted through the zein core, leading to the high contrast difference between core and sheath. ${ }^{20,21}$ TEM analysis demonstrated that the core layer was completely encapsulated by the outer layer. As depicted in Fig. 4, the results of SEM showed that the core-shell structure nanofibers had relatively good morphology and the nanofibers were quite uniform and continue with no bead formation. The diameter of the resulting coaxial electrospun nanofibers ranged between 250 and $830 \mathrm{~nm}$, with an average diameter size of $560 \mathrm{~nm}$.

The LC and EE are important indexes to evaluate the quality of encapsulation. It was found that the LC and EE of fish oil in the coaxial electrospun nanofibers were $14.5 \%$ and $96.9 \%$, respectively. These results indicated that the fish oil can be efficiently encapsulated by coaxial electrospinning.

\subsection{Characterization of coaxial electrospun nanofibers}

FTIR spectroscopy has been frequently used to investigate the basic structure, the compatibility among the components and the second-order interactions such as hydrogen bonding, electrostatic interactions of material. The FTIR spectra of zein, fish
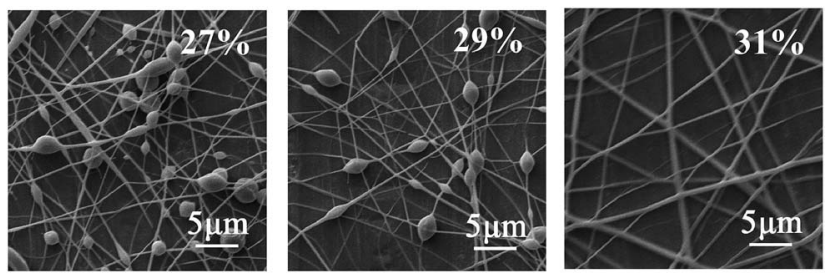

Fig. 2 SEM images of the PVP nanofibers at different PVP concentration. 

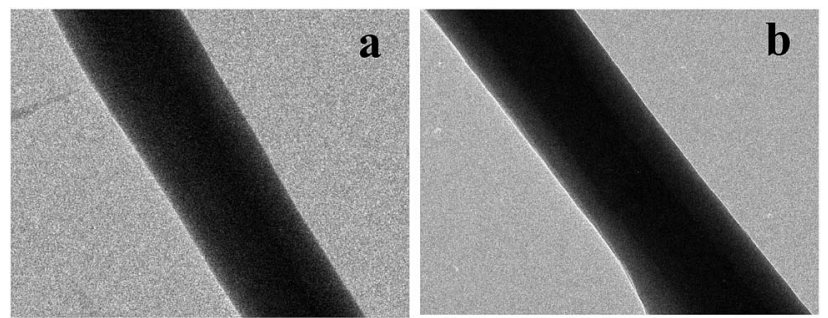

Fig. 3 TEM image of single (a) and coaxial (b) electrospun nanofiber.

oil, PVP and coaxial nanofibers were depicted in Fig. 5. For fish oil, the peak at $3013 \mathrm{~cm}^{-1}$ was assigned to the $=\mathrm{C}-\mathrm{H}$ stretching of double bonds and the peak at $1738 \mathrm{~cm}^{-1}$ was assigned to the $\mathrm{C}=\mathrm{O}$ stretching of carboxyl groups. ${ }^{22}$ These absorptions play an important role in identifying fish oil. Two well-defined sharp peaks at 1655 and $1534 \mathrm{~cm}^{-1}$ were observed for zein. The former showed the $\mathrm{C}=\mathrm{O}$ stretching vibration of amide I, whereas the latter was attributed to the $\mathrm{N}-\mathrm{H}$ bending vibration. ${ }^{23}$ The dominant peak of PVP appeared at $1660 \mathrm{~cm}^{-1}$ $\left(\mathrm{C}=\mathrm{O}\right.$ stretching), and $2955 \mathrm{~cm}^{-1}$ (C-H stretching). ${ }^{24}$ After coaxial electrospinning, the peaks at 3013 and $1738 \mathrm{~cm}^{-1}$ still existed, suggesting that fish oil was successfully encapsulated in the coaxial nanofibers. The amide I band was displaced to $1658 \mathrm{~cm}^{-1}, \mathrm{~N}-\mathrm{H}$ indicator peak was shifted to higher wave number at $1538 \mathrm{~cm}^{-1}$ and the $\mathrm{C}-\mathrm{H}$ stretching indicator peak was shifted to $2962 \mathrm{~cm}^{-1}$. These phenomena indicated that there existed secondary interactions involving electrostatic and hydrophobic interactions among fish oil, zein and PVP, which could improve the compatibility of coaxial electrospun nanofibers.

The core-shell structure nanofibrous mat was further characterized by TGA to investigate its thermal stability. The results of TGA and their respective derivative thermogravimetric analysis (DTG) of zein, fish oil, PVP and coaxial electrospun nanofibers from 40 to $700{ }^{\circ} \mathrm{C}$ were shown in Fig. 6 . The temperature which gives the highest rate of weight loss in the DTG curve is generally taken as degradation temperature $\left(T_{\mathrm{d}}\right)$ of the compound. ${ }^{25}$ It was found that there was no significant difference $(p>0.05)$ between the $T_{\mathrm{d}}$ values of pure zein $\left(326{ }^{\circ} \mathrm{C}\right)$ and zein in the coaxial nanofibers $\left(327^{\circ} \mathrm{C}\right)$. A similar phenomenon was also observed for the pure PVP (432 ${ }^{\circ} \mathrm{C}$ ) and the PVP in the coaxial nanofibers $\left(434{ }^{\circ} \mathrm{C}\right)$. However, it
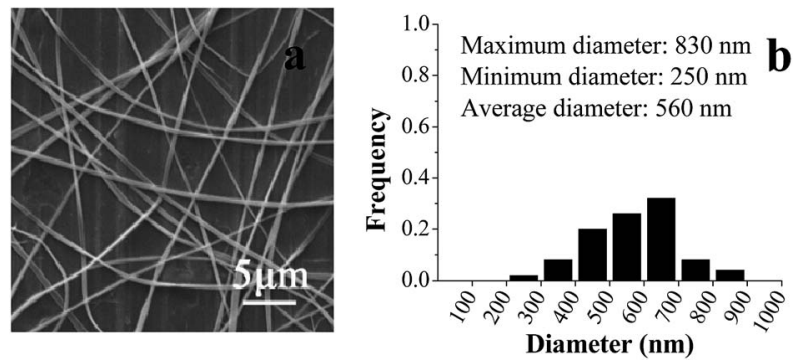

Fig. 4 SEM image (a) and fiber diameter distribution (b) of the coaxial electrospun nanofibers.

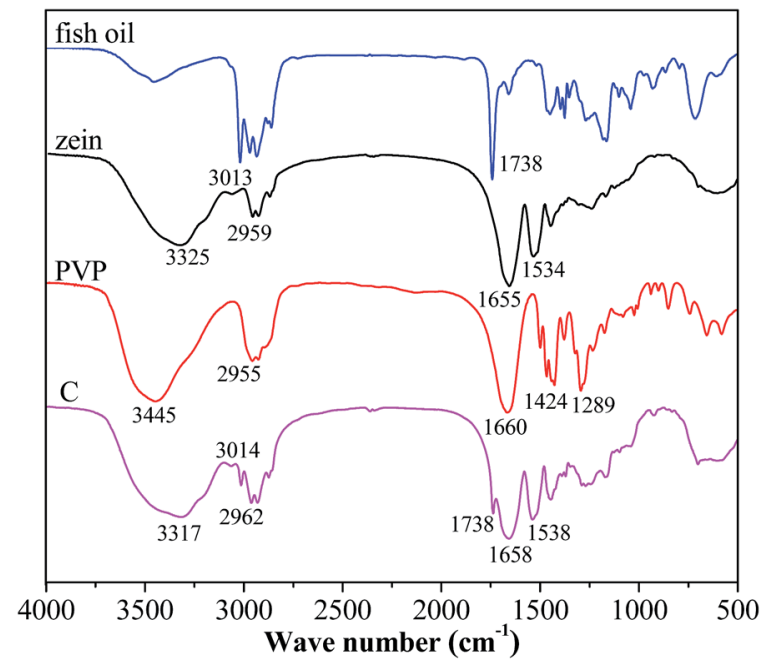

Fig. 5 FTIR spectra of different samples (C: coaxial electrospun nanofibers).

is worth noting that the $T_{\mathrm{d}}$ of fish oil in the coaxial electrospun nanofibers was shifted to higher temperature $\left(302{ }^{\circ} \mathrm{C}\right)$ compared with pure fish oil $\left(256{ }^{\circ} \mathrm{C}\right)$ and this change was significant $(p<0.05)$. These results indicated that coaxial electrospinning effectively enhanced the thermal stability of encapsulated fish oil.

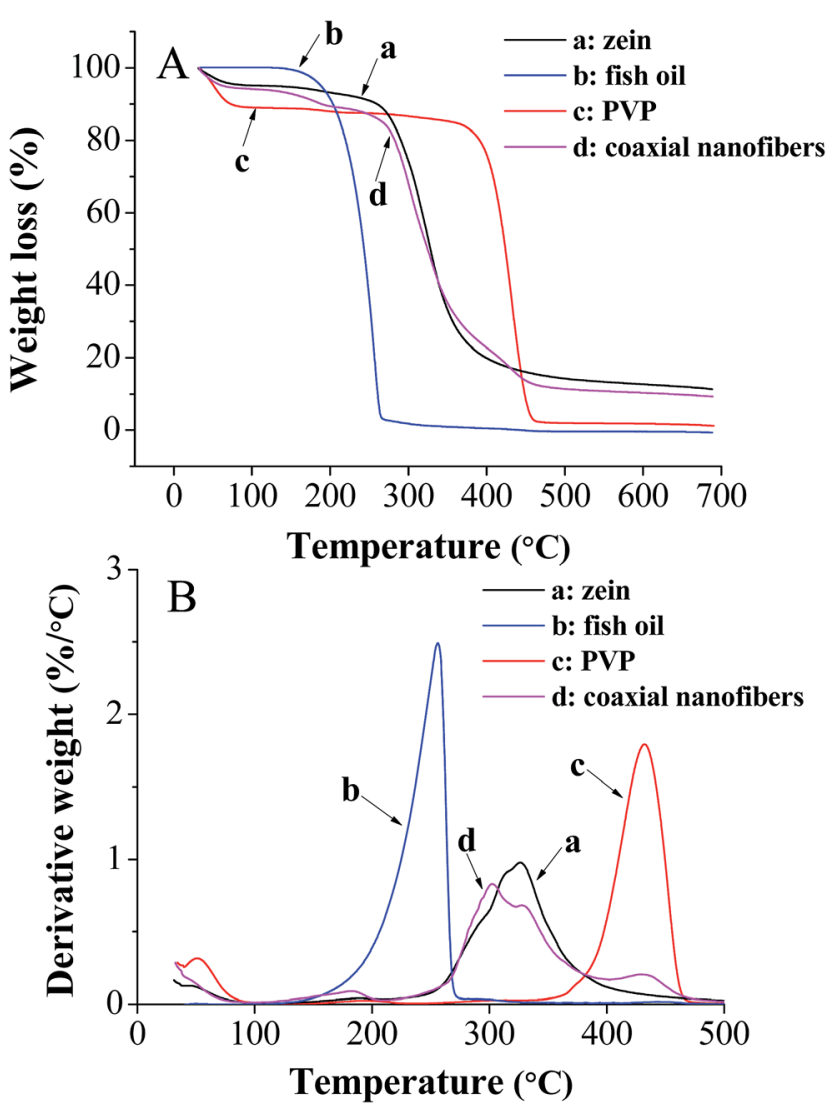

Fig. 6 TGA (A) and DTG (B) of different samples. 


\subsection{Oxidative stability of encapsulated fish oil}

The oxidation of fish oil would create a variety of undesirable ingredients, such as free radicals and hydroperoxides, thus resulting in fat deterioration. ${ }^{26}$ The peroxide value (PV) of lipid is a standard index to determine the deterioration degree of fat and oil. Hence, the PV of encapsulated fish oil in the coaxial nanofibrous mat was measured in the absence or presence of oxygen at 25,45 and $60{ }^{\circ} \mathrm{C}$. The PV of non-encapsulated fish oil was analyzed as the control. Additionally, the PV of encapsulated fish oil in the single nanofibrous mat was also investigated to understand the difference between these two electrospinning processes in the enhancement of oxidative stability of encapsulated fish oil.

Fig. 7 depicted the development of PVs of unencapsulated and encapsulated fish oils under anaerobic and aerobic conditions at different temperature. It was found that under anaerobic conditions, during 25 day storage, all samples had low PV at $25{ }^{\circ} \mathrm{C}$. While at $45{ }^{\circ} \mathrm{C}$, the PV of each sample increased with time and the PV of encapsulated fish oil was always lower than that of unencapsulated fish oil. This trend was more pronounced at $60{ }^{\circ} \mathrm{C}$. These results indicated that electrospinning is an efficient encapsulation method to protect fish oil against oxidation, and the protective effect enhances with the increase of temperature. Interestingly, for the encapsulated fish oil, the PV of single nanofibers was significantly higher ( $p<$ 0.05) than that of coaxial nanofibers at $45^{\circ} \mathrm{C}$ after storage for 20 days and at $60{ }^{\circ} \mathrm{C}$ after storage for 5 days. There is a clear
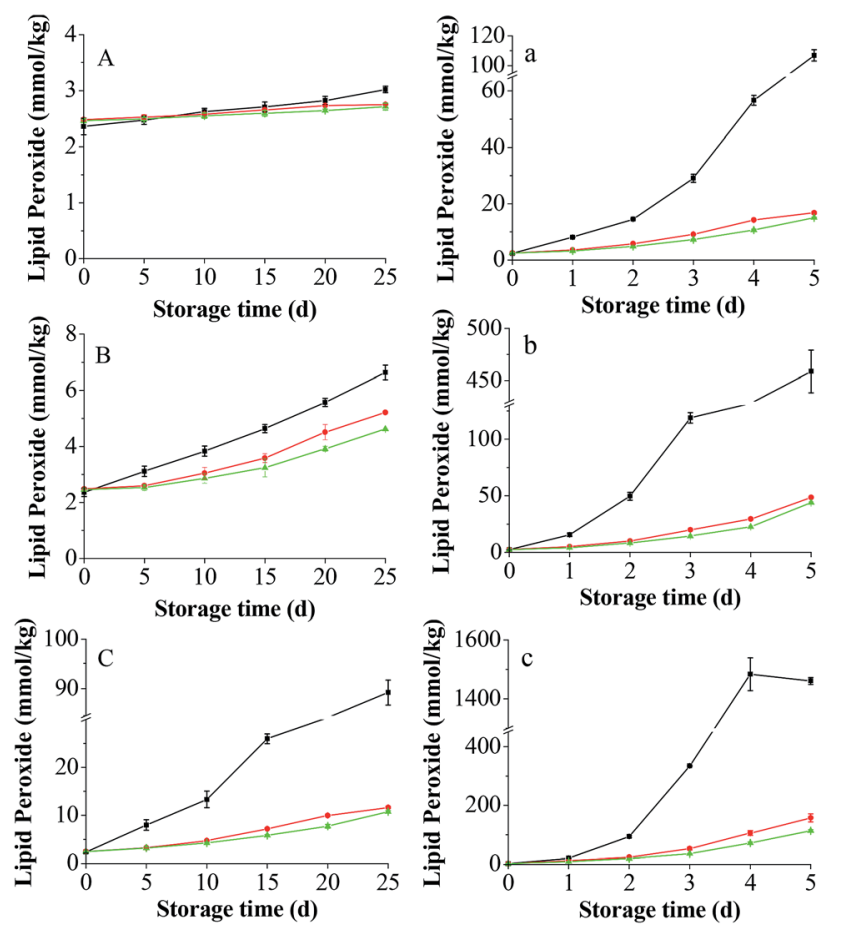

$\rightarrow$ fish oil - - single nanofibers $-\Delta$-coaxial nanofibers

Fig. 7 Development of lipid peroxide of unencapsulated and encapsulated fish oils stored at $25^{\circ} \mathrm{C}(\mathrm{A}, \mathrm{a}), 45^{\circ} \mathrm{C}(\mathrm{B}, \mathrm{b})$ and $60^{\circ} \mathrm{C}(\mathrm{C}, \mathrm{c})$ for a period of 25 days under anaerobic $(A, B, C)$ and aerobic $(a, b, c)$ conditions. difference in the oxidation profile for unencapsulated and encapsulated fish oils under aerobic conditions. Drastic increase in the PV was observed for unencapsulated fish oil at all storage temperatures. Comparatively, the PV of encapsulated fish oil still maintained a slow growth. Moreover, the PV of encapsulated fish oil in the coaxial nanofibers was significantly lower $(p<0.05)$ than that of single nanofibers at all temperatures except storing at $60{ }^{\circ} \mathrm{C}$ for 2 days, indicating that the encapsulated fish oil in the coaxial electrospun nanofibers had greater oxidative stability. The possible reason was that the core-shell structure nanofibrous mat had continuous double layer of nanofibers, and fish oil was encapsulated in the inner layer which made it difficult for oxygen to diffuse and contact with fish oil.

To further understand the enhancing effect of coaxial electrospinning on the oxidative stability of encapsulated fish oil, the oxidation kinetics was investigated. To determine the rate constant of peroxide formation reaction, eqn (1) was fitted to the peroxide values data.

$$
\ln \left(P / P_{0}\right)=k t
$$

where, $P$ is the concentration of oxidation product, $P_{0}$ is the initial value of $P$ at $t=0, k$ is the reaction rate constant, and $t$ is time.

Furthermore, the correlation of the reaction rate constant with temperature follows the Arrhenius equation as shown in eqn (2): ${ }^{27}$

$$
k=k_{0} \times \exp \left(-E_{\mathrm{a}} / R T\right)
$$

where $k$ is the reaction rate constant, $k_{0}$ is the frequency factor, $E_{\mathrm{a}}$ is the activation energy $\left(\mathrm{J} \mathrm{mol}^{-1}\right), R$ is the gas constant $(8.314$ $\mathrm{J} \mathrm{mol}^{-1} \mathrm{~K}^{-1}$ ), and $T$ is the temperature (K).

By taking the natural logarithm of both sides of eqn (2), it changed to eqn (3):

$$
\ln k=\ln k_{0}-E_{\mathrm{a}} / R T
$$

According to eqn (3), a plot of $\ln k$ versus $1 / T$ gives a straight line with a slope of $-E_{\mathrm{a}} / R$, from which the value of activation energy can be determined.

As shown in Table 1, the coefficient of determination values ranged between 0.97 and 0.99 , meaning that the data were satisfactorily adjusted to the model. The $k$ value of encapsulated fish oil was obviously lower than that of unencapsulated counterpart at all the temperatures. Moreover, $k$ value of coaxial nanofibers was always lower than that of single nanofibers. As for $E_{\mathrm{a}}$, it decreased in the order of coaxial nanofibers $>$ single nanofibers $>$ unencapsulated fish oil under both anaerobic and aerobic conditions. All of these fitting results were consistent with PV results, demonstrating again that the encapsulated fish oil in the coaxial electrospun nanofibrous mat had greater oxidative stability than that in the single electrospun nanofibrous mat.

Table 2 summarized the oxidation kinetics models of different samples. The acceptability limit of PV of fish oil was suggested to be $8 \mathrm{mmol} \mathrm{kg}^{-1}$ by Boran et al., ${ }^{28}$ thus the shelf life 
Table 1 Numerical values of the model parameters under anaerobic and aerobic conditions ${ }^{a}$

\begin{tabular}{|c|c|c|c|c|c|c|c|c|}
\hline Sample & \multicolumn{2}{|l|}{$25^{\circ} \mathrm{C}$} & \multicolumn{2}{|l|}{$45^{\circ} \mathrm{C}$} & \multicolumn{2}{|l|}{$60^{\circ} \mathrm{C}$} & $E_{\mathrm{a}} \times 10^{3}\left(\mathrm{~kJ} \mathrm{~mol}^{-1}\right)$ & $R^{2}$ \\
\hline $\mathrm{F}$ & 0.010 & 0.99 & 0.041 & 0.99 & 0.142 & 0.98 & 63.28 & 0.99 \\
\hline S & 0.005 & 0.98 & 0.032 & 0.97 & 0.066 & 0.98 & 64.66 & 0.97 \\
\hline C & 0.004 & 0.99 & 0.026 & 0.96 & 0.059 & 0.99 & 65.00 & 0.98 \\
\hline $\mathrm{F}$ & 0.731 & 0.98 & 1.047 & 0.95 & 1.323 & 0.94 & 14.01 & 0.99 \\
\hline S & 0.405 & 0.98 & 0.597 & 0.98 & 0.798 & 0.95 & 15.93 & 0.99 \\
\hline C & 0.373 & 0.99 & 0.574 & 0.99 & 0.738 & 0.96 & 16.19 & 0.99 \\
\hline
\end{tabular}

${ }^{a}$ F: unencapsulated fish oil, S: encapsulated fish oil in the single nanofibers, C: encapsulated fish oil in the coaxial nanofibers.

of different samples at ambient temperature $\left(20{ }^{\circ} \mathrm{C}\right)$ was calculated according to this level. It was found that under anaerobic condition, the shelf life of unencapsulated fish oil, the encapsulated fish oils in the single nanofibers and coaxial nanofibers were 207, 374 and 439 days, respectively. Obviously, the shelf life of fish oil encapsulated in the coaxial and single electrospun nanofibrous mat were separately improved approximately $110 \%$ and $80 \%$ compared with that of unencapsulated fish oil, indicating that electrospinning is an effective method to encapsulate fish oil. Furthermore, compared to single electrospinning, coaxial electrospinning is a more recommended option to slow down the oxidation process, for the shelf life of encapsulated fish oil in the coaxial nanofibers was 65 days longer than that of single nanofibers.

\subsection{Release behavior of encapsulated fish oil}

3.4.1 Release behavior of encapsulated fish oil in SGF or SIF environment. The release behavior of encapsulated fish oil in gastrointestinal tract plays an important role in the practical application of encapsulation products. The release profile of encapsulated fish oil from coaxial electrospun nanofibers in separate SGF and SIF was depicted in Fig. 8. The release experiment of encapsulated fish oil in the single electrospun nanofibers was also carried out for comparison.

Table 2 Oxidation kinetics models of different samples under anaerobic and aerobic conditions ${ }^{a}$

\begin{tabular}{ll}
\hline Sample & Oxidation kinetics model \\
\hline Anaerobic condition & \\
$\mathrm{F}$ & $k=1.11 \times 10^{9} \times \exp \left(-63.28 \times 10^{3} / R T\right)$ \\
$\mathrm{S}$ & $k=1.04 \times 10^{9} \times \exp \left(-64.65 \times 10^{3} / R T\right)$ \\
$\mathrm{C}$ & $k=1.03 \times 10^{9} \times \exp \left(-65.00 \times 10^{3} / R T\right)$
\end{tabular}

\section{Aerobic condition}

$\begin{array}{ll}\mathrm{F} & k=2.082 \times 10^{2} \times \exp \left(-14.01 \times 10^{3} / R T\right) \\ \mathrm{S} & k=2.488 \times 10^{2} \times \exp \left(-15.92 \times 10^{3} / R T\right) \\ \mathrm{C} & k=2.571 \times 10^{2} \times \exp \left(-16.19 \times 10^{3} / R T\right)\end{array}$

${ }^{a} \mathrm{~F}$ : unencapsulated fish oil, S: encapsulated fish oil in the single nanofibers, C: encapsulated fish oil in the coaxial nanofibers.
In SGF, the total amount of fish oil released from coaxial nanofibers was 53.9\%. After addition of pepsin, the release amount clearly increased, and the corresponding value was $75.9 \%$. Similarly, in SIF, the release study indicated that $45.1 \%$ of encapsulated fish oil was released in $300 \mathrm{~min}$ in the free enzyme solution, while $54.0 \%$ of encapsulated fish oil was released from coaxial nanofibers at the end of the experiment in the enzyme solution. As for single electrospun nanofibers, the release trend was similar to that of coaxial electrospun nanofibers in all simulated fluids, but the release amount of fish oil was slightly higher. These results showed that enzyme has significant effect $(P>0.05)$ on the release behavior.

In order to further understand the release kinetics and mechanism of encapsulated fish oil, the release kinetics of encapsulated fish oil in the nanofibers was investigated. The data were fitted to Korsmeyer-Peppas model as shown in eqn (4):

$$
M_{t} / M_{\infty}=k_{\mathrm{r}} t^{n}
$$
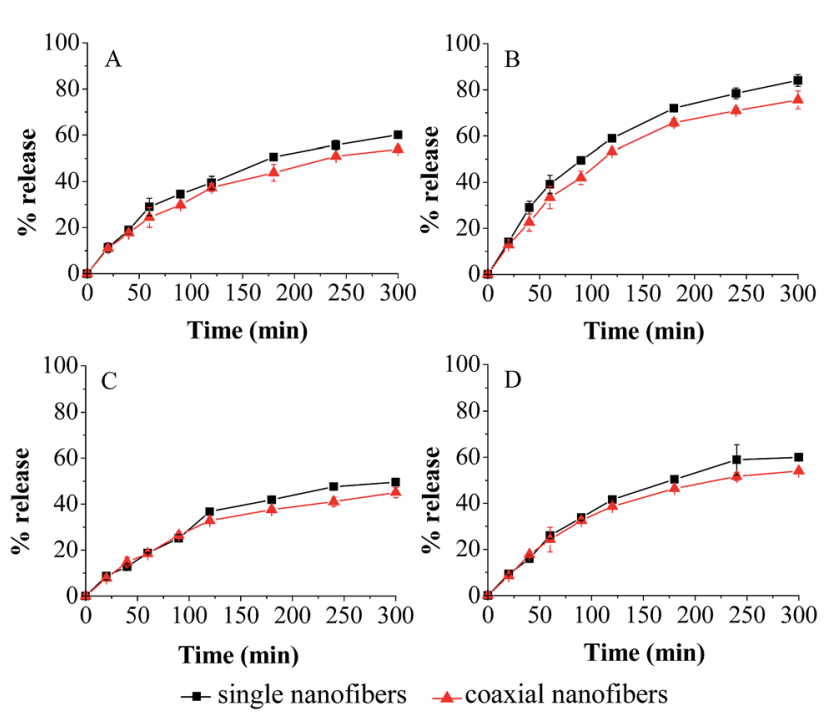

Fig. 8 Release profiles of encapsulated fish oil under simulated gastrointestinal fluids. (A) SGF at pH 1.2, (B) SGF at pH 1.2 in the presence of pepsin, (C) SIF at $\mathrm{pH} 6.8$ and (D) SIF at $\mathrm{pH} 6.8$ in the presence of trypsin. 
where $M_{t} / M_{\infty}$ is the fraction of fish oil released at time $t, k$ is the kinetic constant, $n$ is the release exponent. Using the $n$ and $k$ values derived from eqn (4), the mean dissolution time (MDT) value can be calculated from eqn (5):

$$
\operatorname{MDT}=[n /(n+1)] k_{\mathrm{r}}^{-1 / n}
$$

The parameter MDT has been used not only to describe dissolution and residence profiles, but also to facilitate the comparison of different profiles. ${ }^{29}$

The kinetics parameters of coaxial electrospun nanofibrous mat were calculated from eqn (4) and (5) and presented in Table 3. They all have a relatively high coefficient of determination $\left(0.99 \geq R^{2} \geq 0.96\right)$, indicating that they were satisfactorily adjusted to the model. The release exponent $n$ indicates the release mechanism of encapsulation system. It was found that all of $n$ of coaxial electrospun nanofibers were between 0.59 and 0.70 , suggesting that the release behavior of encapsulated fish oil from the core-shell structure nanofibers was generally controlled by a combination of diffusion and macromolecular chain relaxation. The same consequence was obtained for single electrospun nanofibers. As for the result of MDT, it was found that the MDT of single nanofibers was shorter than that of coaxial nanofibers in all simulated fluids, which was consistent with the result of release profile. Obviously, coaxial electrospinning slightly decreased the release amount of encapsulated fish oil compared with single electrospinning. This might be due to the larger diameter of coaxial nanofibers which resulted in smaller overall specific surface area, and thus influence the release behavior of encapsulated fish oil in the coaxial electro-spun nanofibrous mat.

3.4.2 Release behavior of encapsulated fish oil in sequential SGF and SIF treatment. In order to make the release experiment more similar to the authentic human digestive environment, single and coaxial electrospun nanofibrous mats were sequential exposed to the simulated gastrointestinal tract fluids in the absence or presence of enzymes.

As shown in Fig. 9, in the absence of enzymes, after $2 \mathrm{~h}$ gastric stage, $37.3 \%$ and $34.8 \%$ fish oil was released from single and coaxial nanofibers, respectively. And at the end of the gastrointestinal stage, the amount of released fish oil was $74.8 \%$ and $66.6 \%$, separately. Similar to the above-mentioned results in the separate SGF and SIF environment, enzymes remarkably increased the release amount of encapsulated fish oil in two nanofibrous mats. The cumulative release of fish oil from the single and coaxial nanofibers was respective 58.1 and $52.3 \%$ (in SGF $2 \mathrm{~h}$ ), and 33.2 and $31.1 \%$ (in SIF $4 \mathrm{~h}$ ) in the presence of enzymes. Although the coaxial electrospinning slightly reduced the release amount of encapsulated fish oil, the cumulative release amount of fish oil was almost up to $85 \%$ in the presence of enzymes, which demonstrated again that coaxial electrospinning is an effective strategy to encapsulate fish oil.
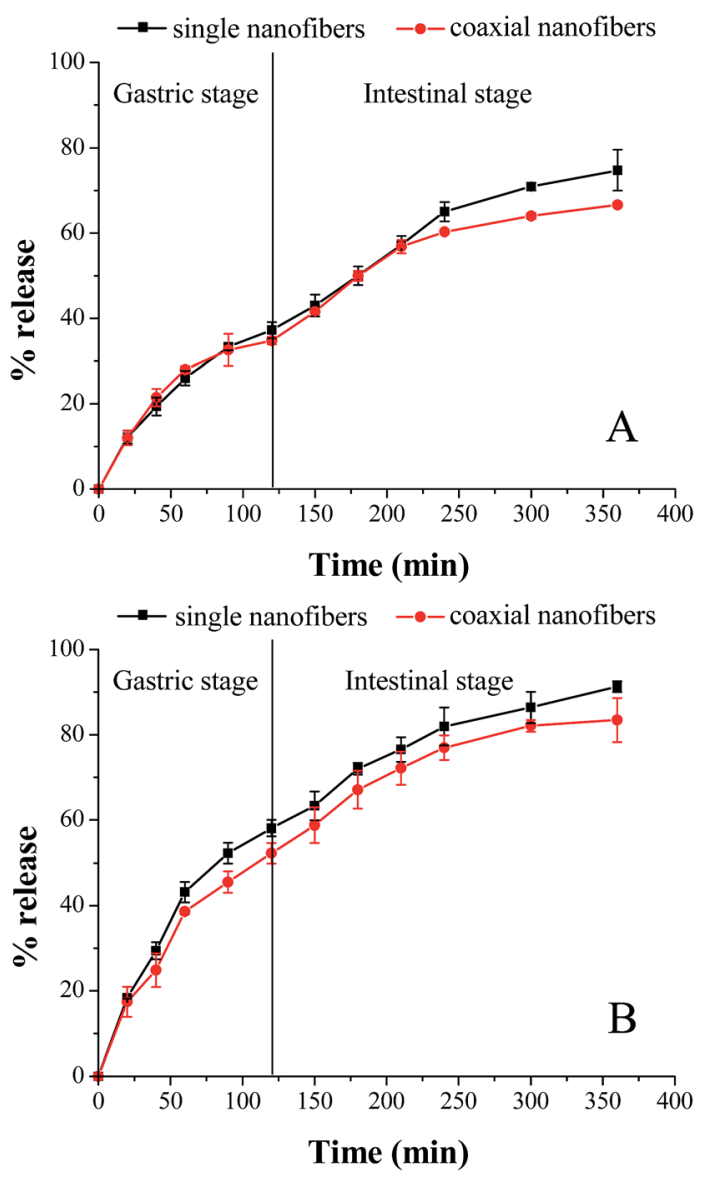

Fig. 9 Release kinetics of fish oil from the single and coaxial nanofibers during an in vitro digestion in the absence (A) and presence (B) of enzymes.

Table 3 Numerical values of the model parameters in various simulated media ${ }^{a}$

\begin{tabular}{|c|c|c|c|c|c|}
\hline Simulated medium & Sample & $n$ & $k_{\mathrm{r}}\left(\min ^{-n}\right)$ & $R^{2}$ & $\begin{array}{l}\text { Mean dissolution } \\
\text { time (min) }\end{array}$ \\
\hline SGF & $\mathrm{S}$ & 0.61 & 0.02 & 0.97 & 231.05 \\
\hline \multirow[t]{2}{*}{ SGF with pepsin } & $\mathrm{S}$ & 0.66 & 0.02 & 0.97 & 149.15 \\
\hline & $\mathrm{C}$ & 0.64 & 0.02 & 0.97 & 176.19 \\
\hline SIF & $\mathrm{S}$ & 0.69 & 0.01 & 0.98 & 323.23 \\
\hline & $\mathrm{C}$ & 0.67 & 0.01 & 0.96 & 387.64 \\
\hline
\end{tabular}

${ }^{a}$ S: single electrospun nanofibers, C: coaxial electrospun nanofibers. 


\section{Conclusions}

In this study, fish oil was successfully encapsulated in a composite nanofibrous mat via coaxial electrospinning. The obtained coaxial nanofibers had a clear core-shell structure as demonstrated by TEM analysis and the average diameter of which was $560 \mathrm{~nm}$. The LC and EE of fish oil were $14.5 \%$ and $96.9 \%$, respectively. FTIR spectra revealed that fish oil was successfully embedded into the coaxial electrospun nanofibers and there existed secondary interactions among the molecules of fish oil, zein and PVP. The thermal stability of fish oil was effectively enhanced when it was encapsulated in the coaxial nanofibrous mat. Compared with single electrospinning, coaxial electrospinning significantly improved the oxidative stability of encapsulated fish oil and the shelf life of encapsulated fish oil in the coaxial nanofibers was 65 days longer than that of single nanofibers. The release amount of encapsulated fish oil in the coaxial nanofibers was less than that of single nanofibers $(83.5 \%$ vs. $91.3 \%)$, however, it is expected to be improved by choosing other different film-forming polymer or modifying its structure for constructing shell layer in the future work. These results indicated that coaxial electrospinning is an effective strategy to improve the oxidative stability of encapsulated fish oil, thus it is promising in production of fish oil functional food.

\section{Acknowledgements}

We gratefully acknowledge the National Natural Science Foundation of China (No. 31671852), the Natural Science Foundation of Guangdong Province (No. 2015A030313217), and the National Natural Science Foundation of China (NSFC)Guangdong Joint Foundation Key Project (No. U1501214) for financial support.

\section{References}

1 M.-J. Choi, U. Ruktanonchai, A. Soottitantawat and S.-G. Min, Food Res. Int., 2009, 42, 989-997.

2 P. K. Binsi, N. Nayak, P. C. Sarkar, A. Jeyakumari, P. Muhamed Ashraf, G. Ninan and C. N. Ravishankar, Food Chem., 2017, 219, 158-168.

3 M. Aghbashlo, H. Mobli, A. Madadlou and S. Rafiee, Food Bioprocess Technol., 2012, 6, 1561-1569.

4 P. N. Ezhilarasi, P. Karthik, N. Chhanwal and C. Anandharamakrishnan, Food Bioprocess Technol., 2012, 6, 628-647.

5 S. Singh and H. S. Nalwa, J. Nanosci. Nanotechnol., 2007, 7, 3048-3070.
6 Y. P. Neo, C. O. Perera, M. K. Nieuwoudt, Z. Zujovic, J. Jin, S. Ray and M. Gizdavic-Nikolaidis, J. Agric. Food Chem., 2014, 62, 5163-5172.

7 C. Yang, T. Zhu, J. Wang, S. Chen and W. Li, RSC Adv., 2015, 5, 69423-69429.

8 P. Wen, D. H. Zhu, K. Feng, F. J. Liu, W. Y. Lou, N. Li, M. H. Zong and H. Wu, Food Chem., 2016, 196, 996-1004.

9 Q. Ma, M. Pyda, B. Mao and P. Cebe, Polymer, 2013, 54, 25442554.

10 P. Wen, D. H. Zhu, H. Wu, M. H. Zong, Y. R. Jing and S. Y. Han, Food Control, 2016, 59, 366-376.

11 S. Sahoo, L. T. Ang, J. C. Goh and S. L. Toh, J. Biomed. Mater. Res., Part A, 2010, 93, 1539-1550.

12 G. Ozaydin-Ince, K. K. Gleason and M. C. Demirel, Soft Matter, 2011, 7, 638-643.

13 G. Dogan, G. Basal, O. Bayraktar, F. Ozyildiz, A. Uzel and I. Erdogan, Microsc. Res. Tech., 2016, 79, 38-49.

14 A. Lópezrubio, E. Sanchez, Y. Sanz and J. M. Lagaron, Biomacromolecules, 2009, 10, 2823-2829.

15 K. Moomand and L.-T. Lim, Food Hydrocolloids, 2015, 46, 191-200.

16 K. Moomand and L.-T. Lim, Food Res. Int., 2014, 62, 523-532. 17 C. B. Giller, D. B. Chase, J. F. Rabolt and C. M. Snively, Polymer, 2010, 51, 4225-4230.

18 N. Okutan, P. Terzi and F. Altay, Food Hydrocolloids, 2014, 39, 19-26.

19 A.-C. Vega-Lugo and L.-T. Lim, J. Polym. Sci., Part B: Polym. Phys., 2012, 50, 1188-1197.

20 M. Pakravan, M. C. Heuzey and A. Ajji, Biomacromolecules, 2012, 13, 412-421.

21 D. Han, MRS Online Proc. Libr., 2008, 1094, DOI: 10.1557/ PROC-1094-DD06-02.

22 S. Torres-Giner, A. Martinez-Abad, M. J. Ocio and J. M. Lagaron, J. Food Sci., 2010, 75, N69-N79.

23 S. Torres-Giner, E. Gimenez and J. M. Lagaron, Food Hydrocolloids, 2008, 22, 601-614.

24 D. G. Yu, X. X. Shen, C. Branford-White, K. White, L. M. Zhu and S. W. Bligh, Nanotechnology, 2009, 20, 055104.

25 Y. P. Neo, S. Ray, J. Jin, M. Gizdavic-Nikolaidis, M. K. Nieuwoudt, D. Liu and S. Y. Quek, Food Chem., 2013, 136, 1013-1021.

26 J. Annamalai, K. C. Dushyant and V. Gudipati, J. Food Process. Preserv., 2015, 39, 1944-1955.

27 S. Calligaris, S. D. Pieve, G. Kravina, L. Manzocco and C. M. Nicoli, J. Food Sci., 2008, 73, E51-E56.

28 G. Boran, H. Karaçam and M. Boran, Food Chem., 2006, 98, 693-698.

29 F. Podczeck, Int. J. Pharm., 1993, 97, 93-100. 